\title{
Topical Treatment of Infectious Vaginitis: Effects of Antibiotic, Antifungal and Antiseptic Drugs on the Growth of Normal Vaginal Lactobacillus Strains
}

\author{
Christel Neut ${ }^{1}$, François Verrière ${ }^{2 *}$, Hans J. Nelis ${ }^{3}$, Tom Coenye ${ }^{3}$ \\ ${ }^{1}$ Laboratoire de Bactériologie Clinique, Faculté de Pharmacie, Lille, France \\ ${ }^{2}$ Laboratoire Innotech International, Arcueil, France \\ ${ }^{3}$ Laboratorium voor Farmaceutische Microbiologie, Gent, Belgium \\ Email: christel.neut@univ-lille2.fr, ${ }^{*}$ francois.verriere@innothera.com, tom.coenye@ugent.be, \\ hans.nelis@ugent.be
}

Received 24 February 2015; accepted 23 March 2015; published 25 March 2015

Copyright (C) 2015 by authors and Scientific Research Publishing Inc.

This work is licensed under the Creative Commons Attribution International License (CC BY).

http://creativecommons.org/licenses/by/4.0/

(c) (i) Open Access

\section{Abstract}

Objectives: The balance of the vaginal ecosystem depends on lactobacilli whose biofilm protects against microorganisms that are not normally present or are subdominant in vaginal flora. Vaginal infection treatments should eliminate pathogens while preserving the Lactobacillus flora to prevent relapse or recurrence. The objective of this study was to determine the sensitivity of lactobacilli to antiseptics, antibiotics and antifungal agents used, alone or in combination, in the topical treatment of infectious vaginitis. Materials and Methods: The minimum inhibitory concentration (MIC) of 15 active ingredients and the minimum inhibitory dilution (MID) of 17 pharmaceuticals products were determined for three main Lactobacillus strains (L. crispatus, L. gasseri and $L$. jensenii). Results: The MICs of the antibiotics and antifungal agents were above their critical concentrations in the three strains, contrary to the antiseptics. According to their MID, some pharmaceutical products, especially combinations, inhibit the growth of one or more strains at concentrations that can be found in the vaginal mucosa after application at the usual dosage. Conclusions: Certain topical anti-infective drugs may interfere with the growth of lactobacilli, worsening the vaginal flora imbalance. Maintaining vaginal flora balance should be a selection criterion when choosing an anti-infective therapy.

\footnotetext{
${ }^{*}$ Corresponding author.
} 
Keywords

Lactobacillus, Infectious Vaginitis, Antiseptics, Antibiotics, Antifungal Agents

\section{Introduction}

Skin and mucous membranes are constantly colonized by microorganisms that form a physiological biofilm. This biofilm protects against colonization by exogenous potential pathogens [1] [2]. In the vaginal epithelium, further protection is provided by lactic acid produced by Lactobacillus bacteria [1] [2]. Lactate accumulation maintains the vaginal $\mathrm{pH}$ between 3.8 and 4.5, where few pathogens are able to develop. This protects the vaginal cavity from colonization, particularly by fecal bacteria such as Enterobacteriaceae, Enterococci and Clostridium, and therefore protects against vaginal, uterus or urinary tract infections [3]-[5]. In addition, a number of Lactobacillus strains produce hydrogen peroxide [6]-[8]. This bactericidal agent plays a key role in regulating vaginal flora by inhibiting pathogens. Ultimately, the presence of lactobacilli is fundamental for vaginal physiology. Lactobacillus gasseri, Lactobacillus jensenii and Lactobacillus crispatus are the strains producing hydrogen peroxide most frequently isolated in normal vaginal flora [9] [10].

Changes to the vaginal environment (change in $\mathrm{pH}$, douching, etc.) can create an imbalance in the physiological flora and cause microorganisms that are not normally present or subdominant to thrive, resulting in infectious vaginitis [11] [12]. Based on the colonizing microorganism, the infectious vaginitis is classified as fungal (mainly Candida), Trichomonas (parasite), bacterial or mixed (several microorganisms). Various topical treatments can be considered to treat infectious vaginitis, depending on the microbial infection: an antifungal agent, an antiseptic, an antibiotic, an antiparasitic or a combination of more than one of these active ingredients in a vaginal formulation (pessaries, capsules, tablets, suppositories). Unlike antiseptics, which have a broad spectrum activity on all microorganisms, antibiotics specifically target bacteria and have a fairly narrow spectrum of activity. Around $25 \%$ of cases are mixed vaginitis, warranting the use of topical antimicrobial combinations such as antifungal agents and antibiotics [13].

It is acknowledged that oral antibiotics can lead to vaginal mycosis [14]. Thus, $3 \%$ to $20 \%$ of patients with idiopathic vulvovaginal candidiasis recently took oral antibiotics; the longer the treatment duration the greater the risk of candidiasis [14]-[16]. The change in Lactobacillus flora could explain candidiasis induced by antibiotics, given that an inverse correlation between the presence of protective lactobacilli in vaginal flora and vaginitis development can be observed [17]. Furthermore, it has been demonstrated that recolonization by hydrogen peroxide-producing lactobacilli one month after treatment of genital infections only occurs in $40 \%$ of patients when oral metronidazole is taken and in 57\% when topical clindamycin is used [18]. However, the effect of antimicrobial agents used to treat vaginitis on the balance of Lactobacillus flora has rarely been assessed.

In this in vitro study, the effect of topical treatments of infectious vaginitis was tested on the growth of Lactobacillus strains. The objective was to identify the active ingredients and formulations potentially less harmful to the balance of normal vaginal flora.

\section{Materials and Methods}

\subsection{Active Ingredients, Pharmaceutical Products and Bacterial Cultures}

The antimicrobial active ingredients tested were obtained from Sigma (Bornem, Belgium). Innotech International Laboratory (Arcueil, France) provided the 17 vaginal formulations tested comprising 15 of the most widely used active ingredients in Europe (azole derivatives, nystatin, antibiotics, antiseptics, etc.).

The activity of the different products (Table 1) was evaluated for three strains of Lactobacillus: L. crispatus (strain LMG 11440), L. gasseri (strain LMG 13134) and L. jensenii (strain LMG 6414). The strains were obtained from the BCCM/LMG Bacteria Collection (Ghent, Belgium) and were grown on MRS agar (Oxoid, Drongen, Belgium) at $37^{\circ} \mathrm{C}$.

\subsection{Determination of the Minimum Inhibitory Concentration (MIC) of the Pure Active Ingredients}

The active ingredients were tested separately and the minimum inhibitory concentration (MIC) was assessed us 
Table 1. Pharmaceutical products tested $(n=17)$ and their active ingredients.

\begin{tabular}{|c|c|c|c|}
\hline Vaginal formulation & Active ingredients & Quantity per unit & Quantity (mg/mL) \\
\hline \multicolumn{4}{|l|}{ Antiseptics } \\
\hline \multirow[t]{2}{*}{$\mathrm{CP}$} & Chlorquinaldol & $200 \mathrm{mg}$ & 40 \\
\hline & Promestriene & $10 \mathrm{mg}$ & 2 \\
\hline G & Chlorhexidine gluconate & $16 \mathrm{mg}$ & 3.2 \\
\hline $\mathrm{P}$ & Povidone-iodine & $250 \mathrm{mg}$ & 5 \\
\hline \multicolumn{4}{|l|}{ Antifungal agents } \\
\hline $\mathrm{C}$ & Clotrimazole & $200 \mathrm{mg}$ & 40 \\
\hline $\mathrm{F} 4$ & Fenticonazole & $200 \mathrm{mg}$ & 40 \\
\hline F12 & Fenticonazole & $600 \mathrm{mg}$ & 120 \\
\hline $\mathrm{K}$ & Ketoconazole & $400 \mathrm{mg}$ & 80 \\
\hline M & Miconazole & $400 \mathrm{mg}$ & 80 \\
\hline $\mathrm{N}$ & Natamycin & $100 \mathrm{mg}$ & 20 \\
\hline \multicolumn{4}{|l|}{ Antibiotics/Antifungal agents } \\
\hline \multirow[t]{2}{*}{ CK } & Clindamycin & $100 \mathrm{mg}$ & 20 \\
\hline & Ketoconazole & $400 \mathrm{mg}$ & 80 \\
\hline \multirow[t]{2}{*}{ MM } & Metronidazole & $100 \mathrm{mg}$ & 20 \\
\hline & Miconazole & $100 \mathrm{mg}$ & 20 \\
\hline \multirow[t]{5}{*}{ MMPN } & Metronidazole & $400 \mathrm{mg}$ & 80 \\
\hline & Miconazole & $100 \mathrm{mg}$ & 20 \\
\hline & Polymyxin B & $5 \mathrm{mg}$ & 1 \\
\hline & Neomycin & $45 \mathrm{mg}$ & 9 \\
\hline & Centella asiatica & $15 \mathrm{mg}$ & 3 \\
\hline \multirow[t]{3}{*}{ MNN } & Metronidazole & $500 \mathrm{mg}$ & 100 \\
\hline & Neomycin & $65,000 \mathrm{IU}$ & 13 \\
\hline & Nystatin & $100,000 \mathrm{IU}$ & 20 \\
\hline \multirow[t]{3}{*}{ NNP } & Nystatin & $100,000 \mathrm{IU}$ & 20 \\
\hline & Neomycin & $35,000 \mathrm{IU}$ & 7 \\
\hline & Polymyxin B & $35,000 \mathrm{IU}$ & 7 \\
\hline \multirow[t]{3}{*}{ ONN } & Ornidazole & $500 \mathrm{mg}$ & 100 \\
\hline & Neomycin & $100 \mathrm{mg}$ & 20 \\
\hline & Nystatin & $100,000 \mathrm{IU}$ & 20 \\
\hline \multirow[t]{3}{*}{ TNN } & Ternidazole & $200 \mathrm{mg}$ & 40 \\
\hline & Neomycin & $100 \mathrm{mg}$ & 20 \\
\hline & Nystatin & $100,000 \mathrm{IU}$ & 20 \\
\hline \multicolumn{4}{|c|}{ Antiparasitic/Antifungal agent } \\
\hline \multirow[t]{2}{*}{ NN } & Nifuratel & $500 \mathrm{mg}$ & 100 \\
\hline & Nystatin & $200,000 \mathrm{IU}$ & 40 \\
\hline
\end{tabular}


ing a standardized method by microplate dilution (EUCAST method). After culturing for 24 hours, $10 \mathrm{~mL}$ of the Lactobacillus suspension were centrifuged then suspended in $100 \mathrm{~mL}$ of double strength MRS broth. $0.1 \mathrm{~mL}$ of this bacterial suspension was placed in each well of a 96-well microplate. $0.1 \mathrm{~mL}$ of the appropriate dilution of each antimicrobial active ingredient was then added. The microplates were incubated at $37^{\circ} \mathrm{C}$ for 24 hours and the optical density was then read at $490 \mathrm{~nm}$ (Perkin Elmer, Vilvoorde, Belgium). The concentration of the first dilution without Lactobacillus bacterial growth is the minimum inhibitory concentration (MIC) of the active ingredient tested. This MIC should be compared to the critical concentrations (CC) that the Antibiogram Committee of the French Society for Microbiology (SFM) defines each year for antibiotics on the market [19]. The lower and upper CCs of an antibiotic represent the minimum and maximum antibiotic doses that are effective on the bacterial strain and safe for humans. After antibiotic treatment at the usual dose, the concentration of active ingredient which can be obtained in the serum and tissues is expected to range between the lower and upper CCs. A bacterial strain is characterized as sensitive or resistant to a particular antibiotic, based on whether the MIC of the antibiotic is below the lower CC or above the upper CC.

\subsection{Determination of the Minimum Inhibitory Dilution (MID) of the Vaginal Formulations}

The activity of vaginal products, some of which contain more than one active ingredient, was tested according to the procedure used for active ingredients. Each unit (pessary, vaginal capsule, vaginal tablet, vaginal suppository) of vaginal formulation was dissolved in $5 \mathrm{~mL}$ of saline solution (stock solution) for 1 hour at $37^{\circ} \mathrm{C}$. Serial dilutions of factor 2 of the stock solution were made and $0.1 \mathrm{~mL}$ of each dilution was added to the Lactobacillus suspensions prepared in the microplates as described above. The vaginal formulations were diluted from $1 / 2$ ( $50 \%$, the highest concentration) to $1 / 1000$ ( $0.1 \%$, the lowest concentration tested). The minimum inhibitory dilution (MID) of all formulations was achieved for the three Lactobacillus strains and the concentration of each active ingredient corresponding to the MID of the formulations tested was calculated.

\subsection{Estimation of the Influence of Vaginal Formulations on the Growth of Lactobacilli}

To estimate the effect of the vaginal formulations on lactobacilli, a critical MID threshold was defined. This threshold corresponds to the assumed vaginal concentration of the active ingredient(s) after topical administration of the formulation. A vaginal formulation will therefore be considered as harmful to Lactobacillus flora if the MID obtained is at or below the critical threshold. Conversely, a formulation will be considered not to harm lactobacilli if the MID is above the critical threshold. According to the literature, the tissue concentration of the active ingredients found after topical application is generally lower than the concentration corresponding to a 1/100 dilution of the vaginal formulation, but greater than the concentration corresponding to a 1/1000 dilution [20]-[24]. The critical MID threshold was therefore established as a 1/128 dilution, or $0.8 \%$ of the stock solution.

\section{Results}

Table 1 describes the 17 pharmaceutical products tested with their active ingredients, unit dose and concentration. Eight of these products are combinations of antibiotics and antifungal agents or antifungal agents and antiparasitics. The other nine products contain only one active ingredient, either an antifungal agent $(n=6)$ or antiseptic $(\mathrm{n}=3)$.

The minimum inhibitory concentration (MIC) of the 15 active ingredients of the formulations tested was determined for each of the three strains of lactobacilli (Table 2). Chlorhexidine and Polyvinylpyrrolidone (PVP), antiseptic active ingredients, have low MICs for the three strains of Lactobacillus, well below the critical concentrations (CC) established for these active ingredients. Conversely, for all the antibiotics and antifungal agents tested, the MIC values are high, above the CCs.

Table 3 summarizes the MID of the 17 pharmaceutical products for the three Lactobacillus tested strains and assesses their impact on Lactobacillus growth. Overall, the MID values of the formulations are consistent with the MICs of their active ingredients (Table 2). For example, for the Nystatin-Neomycin-Polymyxin B combination (NNP), the concentrations of the three active ingredients at the MID are consistent with the MICs calculated for the pure active ingredients (between 110 and $440 \mathrm{mg} / \mathrm{L}$ for polymyxin B and neomycin, and between 310 
Table 2. MICs of pure active ingredients $(\mathrm{n}=15)$ for the 3 Lactobacillus strains.

\begin{tabular}{|c|c|c|c|c|}
\hline & & \multicolumn{3}{|c|}{ Minimum inhibitory concentration } \\
\hline \multicolumn{2}{|c|}{ Active ingredient } & L. crispatus & L. gasseri & L. jensenii \\
\hline \multirow[t]{2}{*}{ Antiseptics } & Chlorhexidine (\%) & 0.04 & 0.01 & 0.02 \\
\hline & Polyvinylpyrrolidone (povidone) (\%) & 0.078 & 0.078 & 0.078 \\
\hline \multirow[t]{6}{*}{ Antifungal agents } & Clotrimazole (mg/L) & $>4$ & $>4$ & $>4$ \\
\hline & Econazole (mg/L) & $>4$ & $>4$ & $>4$ \\
\hline & Ketoconazole (mg/L) & $>4$ & $>4$ & $>4$ \\
\hline & Miconazole (mg/L) & $>4$ & $>4$ & $>4$ \\
\hline & Natamycin (mg/L) & $>512$ & $>512$ & $>512$ \\
\hline & Nystatin (mg/L) & $>16$ & $>16$ & $>16$ \\
\hline \multirow[t]{6}{*}{ Antibiotics } & Chloramphenicol (mg/L) & 8 & 8 & 8 \\
\hline & Clindamycin (mg/L) & $>8$ & $>8$ & $>8$ \\
\hline & Metronidazole (mg/L) & $>512$ & $>512$ & $>512$ \\
\hline & Neomycin (mg/L) & 128 & 512 & 128 \\
\hline & Ornidazole (mg/L) & $>16$ & $>16$ & $>16$ \\
\hline & Polymyxin B (mg/L) & 256 & 256 & 256 \\
\hline
\end{tabular}

Table 3. MIDs of pharmaceutical products $(n=17)$ for the 3 Lactobacillus strains.

\begin{tabular}{|c|c|c|c|}
\hline \multirow[b]{2}{*}{ Marketed product } & \multicolumn{3}{|c|}{ Minimum inhibitory dilution (\% dilution of the marketed product) } \\
\hline & L. crispatus & L. gasseri & L. jensenii \\
\hline \multicolumn{4}{|c|}{ Antiseptics } \\
\hline $\mathrm{CP}$ & $50 \%$ & $25 \%$ & $1.56 \%$ \\
\hline $\mathrm{G} \dagger$ & $1.56 \%$ & $0.4 \%$ & $1.56 \%$ \\
\hline $\mathrm{P}$ & $12.5 \%$ & $12.5 \%$ & $6.25 \%$ \\
\hline \multicolumn{4}{|c|}{ Antifungal agents } \\
\hline $\mathrm{C}$ & $12.5 \%$ & $12.5 \%$ & $12.5 \%$ \\
\hline F4 & $25 \%$ & $12.5 \%$ & $25 \%$ \\
\hline F12 & $50 \%$ & $12.5 \%$ & $50 \%$ \\
\hline K & $3.125 \%$ & $6.25 \%$ & $6.25 \%$ \\
\hline $\mathrm{M} \dagger$ & $0.8 \%$ & $3.125 \%$ & $12.5 \%$ \\
\hline $\mathrm{N}$ & $6.25 \%$ & $6.25 \%$ & $6.25 \%$ \\
\hline \multicolumn{4}{|c|}{ Antibiotics/Antifungal agents } \\
\hline $\mathrm{CK} \dagger$ & $0.4 \%$ & $0.2 \%$ & $1.56 \%$ \\
\hline $\mathrm{MM} \dagger$ & $0.1 \%$ & $6.25 \%$ & $0.2 \%$ \\
\hline MMNP & $6.25 \%$ & $6.25 \%$ & $3.125 \%$ \\
\hline $\mathrm{MNN} \dagger$ & $0.8 \%$ & $3.125 \%$ & $12.5 \%$ \\
\hline NNP & $1.56 \%$ & $6.25 \%$ & $3.125 \%$ \\
\hline $\mathrm{ONN} \dagger$ & $0.8 \%$ & $6.25 \%$ & $0.2 \%$ \\
\hline $\mathrm{TNN} \dagger$ & $0.8 \%$ & $3.125 \%$ & $3.125 \%$ \\
\hline \multicolumn{4}{|c|}{ Antiparasitic/Antifungal agent } \\
\hline $\mathrm{NN} \dagger$ & $0.4 \%$ & $0.4 \%$ & $0.2 \%$ \\
\hline
\end{tabular}

CP: chlorquinaldol-promestriene; G: chlorhexidine gluconate; P: povidone iodine; C: clotrimazole; F4: fenticonazole $40 \mathrm{mg} / \mathrm{mL}$; F12: fenticonazole 120 mg/mL; K: ketoconazole; M: miconazole; N: natamycin; CK: clindamycin-ketoconazole; MM: metronidazole-miconazole; MMNP: metronidazolemiconazole-neomycin-polymyxin B-Centella asiatica; MNN: metronidazole-neomycin-nystatin; NNP: nystatin-neomycin-polymyxin B; ONN: ornidazole-neomycin-nystatin; TNN: ternidazole-neomycin-nystatin; NN: nifuratel-nystatin.

$\dagger$ : Vaginal formulations with an MID at or below the critical threshold of $0.8 \%$ for one or more Lactobacillus strains. 
and $1250 \mathrm{mg} / \mathrm{L}$ for nystatin).

Regarding the antiseptics, two out of the three tested products-Povidone iodine and Chlorquinaldol-Promestriene combination (P and CP, respectively) — gave MIDs well above the critical threshold of $0.8 \%$. On the other hand, Chlorhexidine gluconate antiseptic (G) has an MID below the critical threshold for the L. gasseri strain.

The analysis of the vaginal antifungal formulations Clotrimazole, Fenticonazole 40 and $120 \mathrm{mg} / \mathrm{mL}$, Ketoconazole, Miconazole and Natamycin (C, F4, F12, K, M and N, respectively) gave fairly consistent results with a very high MID for the three Lactobacillus strains in five out of the six tested products. Miconazole formulation (M) is the only product with an MID below the critical threshold determined for one strain (L. crispatus).

As for the antibiotic/antifungal and antiparasitic/antifungal combinations, six out of the eight tested products have MIDs lower than or equal to the critical dilution threshold of $0.8 \%$. Indeed, the MIDs of the MetronidazoleNeomycin-Nystatin and Ternidazole-Neomycin-Nystatin combinations (MNN and TNN, respectively) are $0.8 \%$ for L. crispatus. The pharmaceutical products Metronidazole-Miconazole (MM), Ornidazole-Neomycin-Nystatin (ONN) and Clindamycin-Ketoconazole (CK) give MIDs below this threshold value for 2 strains, whereas Nifuratel-Nystatin formulation (NN) gives MIDs below $0.8 \%$ for the three strains tested.

\section{Discussion}

This in vitro study shows the effect of 17 pharmaceutical products indicated in the topical treatment of infectious vaginitis and the effect of their active ingredients on 3 main Lactobacillus vaginal strains.

The results suggest that the three Lactobacillus strains tested are resistant to the antibiotic and antifungal active ingredients. However, the antiseptic active ingredients could have a destructive effect on lactobacilli in vivo, since their MICs are lower than the critical concentrations.

It should be noted that the CCs correspond to plasma levels. The results are extrapolated for topical use, with a higher tissue concentration after administration than the plasma concentrations achieved by oral administration. Therefore, the MIDs of the vaginal formulations were determined and a critical threshold of $0.8 \%$ was defined, corresponding to the assumed topical concentration after administration [20]-[24]. This threshold can be used to estimate the formulations potentially harmful to the vaginal flora balance. Hence, of the 17 formulations tested, 8 inhibit the growth of at least one of the three major Lactobacillus strains.

Of the three antiseptics tested, two products (P \& CP) do not affect the growth of the Lactobacillus strains studied. These formulations could be considered for adjunctive treatment of vaginitis.

Regarding products containing only an antifungal agent, their very high MIDs suggest that they do not affect the three strains of Lactobacillus, as expected. However, the product containing Miconazole (M) may inhibit the L. crispatus strain (MID $=0.8 \%$ ). The high concentration of active ingredient could explain this formulation's harmful effect on the growth of lactobacilli.

Regarding combinations (antibiotic/antifungal and antibiotic/antiparasitic), only two out of the eight tested pharmaceutical products do not interfere with in vitro growth of the three Lactobacillus strains. For these two formulations (MMPN and NNP), concentrations 2 - 8 times higher than the assumed vaginal concentration after topical application would be required to inhibit lactobacilli. At the assumed topical concentration, the other six combinations inhibit the growth of one strain (MNN and TNN), two strains (CK, MM and ONN) or even of the three strains (NN) of Lactobacillus. Therefore, one can assume that the use of one of these combinations could interfere with the balance of vaginal flora contrary to Metronidazole-Miconazole-Polymyxin B-Neomycin-Centella asiatica and Nystatin-Neomycin-Polymyxin B specialties (MMPN and NNP, respectively), to which the three Lactobacillus strains are resistant at the assumed topical concentration.

The tolerance of lactobacilli is different depending on whether they grow in the presence of the active ingredient alone or the vaginal formulation. Indeed, results of the formulation MID are not always consistent with their active ingredient MICs. Some formulations appear to have a negative impact on the growth of Lactobacillus while their active ingredient MICs are above the CC. Most vaginal formulations have high doses and, as this study shows, the concentration of the active ingredient of a formulation at the MID can be higher than the MIC of the same active ingredient in its pure form. Thus, the dose administered will be higher than the dose tolerated by the Lactobacillus flora. For example, NNP, MNN and TNN share two active ingredients (neomycin and nystatin) but in NNP, which does not affect the growth of lactobacilli at the threshold concentration, the antibiotic is less concentrated. This difference in concentration may explain, at least in part, the disparity in the impact of these three products on the growth of lactobacilli. 
To our knowledge, no in vitro study had yet tested the effect of so many vaginal formulations $(n=17)$ used in the treatment of infectious vaginitis. Previous studies have suggested harmful effects from active ingredients alone (clindamycin, metronidazole) on the growth of Lactobacillus flora [25] [26].

Our study is subject to the limits applicable to all in vitro studies: the results are predictors of an antibiotic's efficacy but do not necessarily guarantee efficacy in vivo [27]. Thus, there may not be a correlation between the in vitro sensitivity of the lactobacilli and the in vivo impact of the vaginal formulations on all Lactobacillus flora. Nevertheless, this study gives an indication of which vaginal formulations are likely to reduce the Lactobacillus component of vaginal flora, leading to the possibility of the vaginal infection recurring.

\section{Conclusion}

The high concentrations of these vaginal formulations' active ingredients in the vaginal mucosa are capable of inhibiting one or more Lactobacillus strains, with harmful effects on the balance of normal vaginal flora. This study indicates that, of the 17 vaginal formulations indicated to treat vaginitis, six combinations of antibiotic and antifungal agents (out of eight), one antiseptic (out of three) and one antifungal agent (out of the six tested) could interfere with the growth of lactobacilli in healthy vaginal flora. This should be taken into account when choosing first-line topical treatments for infectious vaginitis.

\section{Conflict of Interest}

CN: Paid by Innotech International for analyzing results and writing the manuscript;

FV: Chief Medical Officer, Innotech International;

HJN, TC: Paid by Innotech International for conducting experiments.

\section{Fund}

This study was funded by Innotech International.

\section{References}

[1] Reid, G. (2001) Probiotic Agents to Protect the Urogenital Tract against Infection. The American Journal of Clinical Nutrition, 73, 437S-443S.

[2] Hyman, R.W., Fukushima, M., Diamond, L., Kumm, J., Giudice, L.C. and Davis, R.W. (2005) Microbes on the Human Vaginal Epithelium. Proceedings of the National Academy of Sciences of the United States of America, 102, 7952-7957. http://dx.doi.org/10.1073/pnas.0503236102

[3] Hay, P. (2005) Life in the Littoral Zone: Lactobacilli Losing the Plot. Sexually Transmitted Infections, 81, $100-102$. http://dx.doi.org/10.1136/sti.2003.007161

[4] Dover, S.E., Aroutcheva, A.A., Faro, S. and Chikindas, M.L. (2008) Natural Antimicrobials and Their Role in Vaginal Health: A Short Review. International Journal of Probiotics and Prebiotics, 3, 219-230.

[5] Boskey, E.R., Telsch, K.M., Whaley, K.J., Moench, T.R. and Cone, R.A. (1999) Acid Production by Vaginal Flora in Vitro Is Consistent with the Rate and Extent of Vaginal Acidification. Infection and Immunity, 67, 5170-5175.

[6] Atassi, F. and Servin, A.L. (2010) Individual and Co-Operative Roles of Lactic Acid and Hydrogen Peroxide in the Killing Activity of Enteric Strain Lactobacillus Johnsonii NCC933 and Vaginal Strain Lactobacillus Gasseri KS120.1 against Enteric, Uropathogenic and Vaginose-Associated Pathogens. FEMS Microbiology Letters, 304, 29-38. http://dx.doi.org/10.1111/j.1574-6968.2009.01887.x

[7] Eschenbach, D.A., Davick, P.R., Williams, B.L., Klebanoff, S.J., Young-Smith, K., Critchlow, C.M. and Holmes, K.K. (1989) Prevalence of Hydrogen Peroxide-Producing Lactobacillus Species in Normal Women and Women with Bacterial Vaginosis. Journal of Clinical Microbiology, 27, 251-256.

[8] Mijac, V.D., Dukić, S.V., Opavski, N.Z., Dukić, M.K. and Ranin, L.T. (2006) Hydrogen Peroxide Producing Lactobacilli in Women with Vaginal Infections. The European Journal of Obstetrics \& Gynecology and Reproductive Biology, 129, 69-76. http://dx.doi.org/10.1016/j.ejogrb.2005.11.036

[9] Ravel, J., Gajer, P., Abdo, Z., Schneider, G.M., Koenig, S.S., McCulle, S.L., Karlebach, S., Gorle, R., Russell, J., Tacket, C.O., Brotman, R.M., Davis, C.C., Ault, K., Peralta, L. and Forney, L.J. (2011) Vaginal Microbiome of Reproductive-Age Women. Proceedings of the National Academy of Sciences of the United States of America, 15, 46804687. http://dx.doi.org/10.1073/pnas.1002611107

[10] Martín, R., Soberón, N., Escobedo, S. and Suárez, J.E. (2009) Bacteriophage Induction versus Vaginal Homeostasis: 
Role of $\mathrm{H}(2) \mathrm{O}(2)$ in the Selection of Lactobacillus Defective Prophages. International Microbiology, 12, 131-136.

[11] Zozaya-Hinchliffe, M., Lillis, R., Martin, D.H. and Ferris, M.J. (2010) Quantitative PCR Assessments of Bacterial Species in Women with and without Bacterial Vaginosis. Journal of Clinical Microbiology, 48, 1812-1829. http://dx.doi.org/10.1128/JCM.00851-09

[12] Turovskiy, Y., Sutyak Noll, K. and Chikindas, M.L. (2011) The Aetiology of Bacterial Vaginosis. Journal of Applied Microbiology, 110, 1105-1128. http://dx.doi.org/10.1111/j.1365-2672.2011.04977.x

[13] Bohbot, J.M., Sednaoui, P., Verriere, F. and Achhammer, I. (2012) The Etiologic Diversity of Vaginitis. Gynécologie Obstétrique \& Fertilité, 40, 578-581. http://dx.doi.org/10.1016/j.gyobfe.2011.08.001

[14] Spinillo, A., Capuzzo, E., Acciano, S., De Santolo, A. and Zara, F. (1999) Effect of Antibiotic Use on the Prevalence of Symptomatic Vulvovaginal Candidiasis. American Journal of Obstetrics and Gynecology, 180, 14-17. http://dx.doi.org/10.1016/S0002-9378(99)70141-9

[15] Chen, J.Y., Tian, H. and Beigi, R.H. (2009) Treatment Considerations for Bacterial Vaginosis and the Risk of Recurrence. Journal of Women's Health, 18, 1997-2004. http://dx.doi.org/10.1089/jwh.2008.1088

[16] Corsello, S., Spinillo, A., Osnengo, G., Penna, C., Guaschino, S., Beltrame, A., Blasi, N. and Festa, A. (2003) An Epidemiological Survey of Vulvovaginal Candidiasis in Italy. European Journal of Obstetrics \& Gynecology and Reproductive Biology, 110, 66-72. http://dx.doi.org/10.1016/S0301-2115(03)00096-4

[17] Razzak, M.S., Al-Charrakh, A.H. and Al-Greitty, B.H. (2011) Relationship between Lactobacilli and Opportunistic Bacterial Pathogens Associated with Vaginitis. North American Journal of Medical Sciences, 3, 185-192.

[18] Agnew, K.J. and Hillier, S.L. (1995) The Effect of Treatment Regimens for Vaginitis and Cervicitis on Vaginal Colonization by Lactobacilli. Sexually Transmitted Diseases, 22, 269-273. http://dx.doi.org/10.1097/00007435-199509000-00001

[19] Soussy, C.J. (2012) Antibiogram Committee of the French Society for Microbiology, Recommendations. http://www.sfm-microbiologie.org/UserFiles/files/casfm/CASFM_2012.pdf

[20] Odds, F.C. and MacDonald, F. (1981) Persistence of Miconazole in Vaginal Secretions after Single Applications. Implications for the Treatment of Vaginal Candidosis. British Journal of Venereal Diseases, 57, 400-401.

[21] Mendling, W. and Plempel, M. (1982) Vaginal Secretion Levels after 6 Days, 3 Days and 1 Day of Treatment with 100, 200 and 500 mg Vaginal Tablets of Clotrimazole and Their Therapeutic Efficacy. Chemotherapy, 28, 43-47. http://dx.doi.org/10.1159/000238151

[22] Houang, E.T. and Lawrence, A.G. (1985) Systemic Absorption and Persistence of Tioconazole in Vaginal Fluid after Insertion of a Single 300-mg Tioconazole Ovule. Antimicrobial Agents and Chemotherapy, 27, 964-965. http://dx.doi.org/10.1128/AAC.27.6.964

[23] Jones, R.N., Bale, M.J., Hoban, D. and Erwin, M.E. (1993) In Vitro Antimicrobial Activity of Tioconazole and Its Concentrations in Vaginal Fluids Following Topical (Vagistat-1 6.5\%) Application. Diagnostic Microbiology and Infectious Disease, 17, 45-51. http://dx.doi.org/10.1016/0732-8893(93)90069-J

[24] Eriksson, K., Larsson, P.G., Nilsson, M. and Forsum, U. (2011) Vaginal Retention of Locally Administered Clindamycin. APMIS, 119, 373-376. http://dx.doi.org/10.1111/j.1600-0463.2011.02742.x

[25] Aroutcheva, A., Simoes, J.A., Shott, S. and Faro, S. (2001) The Inhibitory Effect of Clindamycin on Lactobacillus in Vitro. Infectious Diseases in Obstetrics and Gynecology, 9, 239-244. http://dx.doi.org/10.1155/S1064744901000394

[26] Simoes, J.A., Aroutcheva, A.A., Shott, S. and Faro S. (2001) Effect of Metronidazole on the Growth of Vaginal Lactobacilli in Vitro. Infectious Diseases in Obstetrics and Gynecology, 9, 41-45. http://dx.doi.org/10.1155/S1064744901000072

[27] Agence Nationale de Sécurité du Médicament (2011) Guidelines for the Appropriate Use of Antibiotics. http://ansm.sante.fr/Activites/Publicite-pour-les-medicaments/Recommandations-pour-la-publicite-aupres-des-professi onnels-de-sante/Recommandations-pour-la-publicite-aupres-des-professionnels-de-sante/Antibiotiques 